\title{
Nonpalpable testicular pure seminoma with elevated serum alpha-fetoprotein presenting with retroperitoneal metastasis: a case report

Shoichiro Iwatsuki ${ }^{1}$, Taku Naiki ${ }^{1 *}$, Noriyasu Kawai ${ }^{1}$, Toshiki Etani ${ }^{1}$, Keitaro lida ${ }^{1}$, Ryosuke Ando ${ }^{1}$, Takashi Nagai ${ }^{1}$, Atsushi Okada', Keiichi Tozawa', Yosuke Sugiyama² and Takahiro Yasui ${ }^{1}$

\begin{abstract}
Background: Patients with a primary pure seminoma in the testis who have elevated serum alpha-fetoprotein are rare and should be treated as patients with nonseminomatous germ cell tumors. However, nonpalpable testicular tumors in this condition have never been reported. We describe a case of nonpalpable pure testicular seminoma with elevated serum alpha-fetoprotein presenting retroperitoneal metastasis.

Case presentation: A 29-year-old Asian man was referred to our hospital with right flank pain. Computed tomography showed a mass located between his aorta and inferior vena cava, but a testicular tumor was not detected. His serum levels of lactate dehydrogenase, alpha-fetoprotein, and DUPAN-2 were high. Although no tumor or nodule was palpable in his testis, ultrasonography revealed multiple low echoic lesions in his right testicular parenchyma. He was diagnosed with right testicular cancer with retroperitoneal lymph node metastasis and underwent right high orchiectomy. A pathological examination revealed pure seminoma and no nonseminomatous components were found in the specimen. Three courses of induction systemic chemotherapy (cisplatin, etoposide, and bleomycin) normalized his serum alpha-fetoprotein and DUPAN-2 levels. Three additional courses of chemotherapy (etoposide and bleomycin) were performed, and treatment was completed with laparoscopic retroperitoneal lymph node dissection. Pathology of the dissected specimen showed fibrous and necrotic tissue with no viable cells. He is alive without recurrence 54 months after orchiectomy.

Conclusions: We report a case of pure testicular seminoma with elevated serum alpha-fetoprotein and DUPAN-2 presenting retroperitoneal metastasis. We recommend an ultrasound examination of bilateral testes when large retroperitoneal tumors are detected in young men, even if a mass is not palpable in the scrotum.
\end{abstract}

Keywords: Alpha-fetoprotein, DUPAN-2, Testicular tumor, Retroperitoneal lymph node dissection, Seminoma, Burned-out tumor, Case report

\section{Background}

Testicular cancers are categorized into seminomatous and non-seminomatous germ cell tumors (NSGCTs), each with a $50 \%$ incidence. Distinguishing NSGCTs from seminomas is important because the therapeutic strategy is different. Many patients with NSGCT have elevated serum tumor markers, such as lactate dehydrogenase (LDH), alpha-fetoprotein (AFP), and

\footnotetext{
* Correspondence: naiki@med.nagoya-cu.ac.jp; rx-nike@hotmail.co.jp 'Department of Nephro-urology, Graduate School of Medical Sciences, Nagoya City University, 1, Kawasumi, Mizuho-cho, Mizuho-ku, 467-8601 Nagoya, Japan

Full list of author information is available at the end of the article
}

human chorionic gonadotropin (hCG). Since seminoma does not induce AFP production, the guidelines recommend that cases with increased AFP levels be treated as cases of NSGCT $[1,2]$. Because, there is a possibility that a hidden focus of NSGCTs like yolk sac tumor is somewhere present. However, in the literature, few cases have been described of patients with histologically pure seminoma presenting with elevated serum AFP levels [3-5].

We report a case of a 29-year-old Asian man with nonpalpable testicular cancer presenting retroperitoneal lymph node metastasis. Although his testicular histology 
showed pure seminoma at orchiectomy, preoperative serum AFP levels were elevated.

\section{Case presentation}

A 29-year-old Asian man with no remarkable past history was referred to our hospital presenting with right flank pain. He had an episode of right flank pain 2 weeks before the first visit. Computed tomography $(\mathrm{CT})$ revealed a retroperitoneal mass $(52 \times 36 \times 31$ $\mathrm{mm}$ ) located between his aorta and inferior vena cava (Fig. 1a), but a testicular tumor was not detected in his testis. His serum levels of LDH, AFP, and DUPAN-2 were high (327 U/l, $29.6 \mathrm{ng} / \mathrm{ml}$, and higher than measurable range, $1600 \mathrm{U} / \mathrm{ml}$, respectively). Serum levels of other tumor markers, such as hCG, carcinoembryonic antigen, carbohydrate antigen 19-9, and soluble interleukin 2 receptor, were within the normal range $(1.1 \mathrm{mIU} / \mathrm{ml}, 2.7 \mathrm{ng} / \mathrm{ml}, 27.9 \mathrm{U} / \mathrm{ml}$, and $157 \mathrm{U} / \mathrm{ml}$, respectively). Although no tumor or nodule was palpable in either testis, ultrasonography revealed multiple low echoic lesions in his right testicular parenchyma (Fig. 1b). He was diagnosed with right testicular cancer with retroperitoneal metastasis, and underwent right high orchiectomy (Fig. 2a). A pathological examination revealed pure seminoma (Fig. 2b); fibrous foci were diffusely observed (Fig. 2c) and no nonseminomatous components were found in the specimen. Because his preoperative serum AFP levels were high, induction chemotherapy combining cisplatin, etoposide, and bleomycin (BEP), was performed. After three courses, his serum AFP and DUPAN-2 levels were normalized. The volume of his retroperitoneal mass decreased to $15 \times 10 \times 7 \mathrm{~mm}$ after three additional courses of chemotherapy combining etoposide and cisplatin (EP). Finally, laparoscopic retroperitoneal lymph node dissection (RPLND) was performed, and pathology of the dissected specimen showed fibrous and necrotic tissue with no viable cells (Fig. 3d). He is alive without recurrence 54 months after orchiectomy.

\section{Discussion}

Patients with elevated serum AFP levels and a primary pure seminoma are treated as patients with NSGCTs, because some nonseminomatous components are presumed to be present either in the testis or at a metastatic site. Therefore, we treated the current case as NSGCTs, and induction chemotherapy followed by RPLND was performed after orchiectomy as in stage IIB NSGCTs. Peterson et al. [5] reported 42 cases with pure seminoma at orchiectomy and showing elevated serum AFP levels. They performed combination chemotherapy based on platinum reagent followed by RPLND. Viable cancer cells were observed in the retroperitoneal lymph nodes of 15 patients $(37.5 \%)$ with pure seminoma showing elevated serum AFP levels. In general, $16.9 \%$ of patients with stage II or greater pure seminoma without elevated serum AFP levels have viable cancer cells in their retroperitoneal lymph nodes [6]; of the patients with NSGCTs who have retroperitoneal lymph node metastases, $12.2 \%$ have viable cancer cells in their retroperitoneal lymph nodes after chemotherapy [7]. Therefore, pure seminomas with elevated serum AFP levels have a relatively lower sensitivity to chemotherapy than pure seminomas without elevated serum AFP levels or NSGCTs. Fortunately, the pathological examination of the retroperitoneal lymph nodes in our patient did not reveal viable cancer cells; the long-term good prognosis suggests that six courses of BEP and EP chemotherapy were effective in this patient.

DUPAN-2 is a well-known tumor marker for pancreatic, bile duct, and colorectal cancers [8]; in our case, serum DUPAN-2 levels, as well as AFP, were increased before orchiectomy. In the literature, only two cases of embryonal carcinomas with elevated serum DUPAN-2 levels have been reported $[9,10]$. Kamoshida et al. reported immunohistochemical analyses of DUPAN-2 expression using paraffin-embedded specimens of 30 testicular germ cell tumors, and DUPAN-2 was expressed in all of the specimens from nine patients
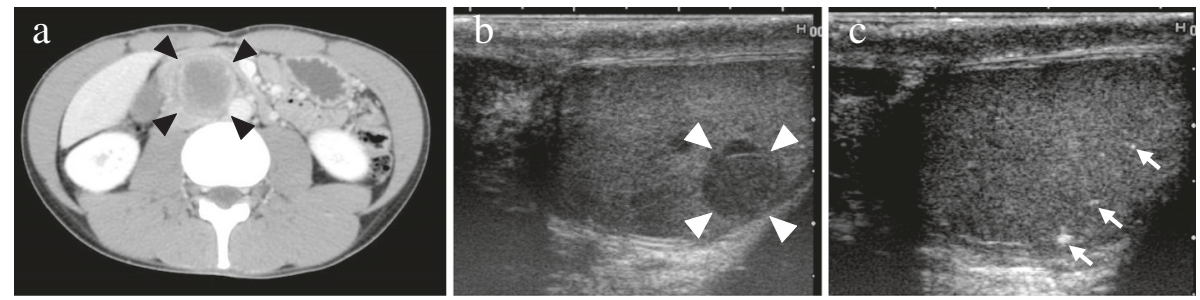

Fig. 1 a Computed tomography shows a retroperitoneal tumor located between the aorta and the inferior vena cava. The size of the tumor (arrowheads) was $52 \times 36 \times 31 \mathrm{~mm}$, and the testicular tumor was not detected in computed tomography. $\mathbf{b}$ Ultrasound examination revealed a tiny low echoic tumor (arrowheads) and a low echoic mosaic pattern around the tumor. c Microcalcifications of various sizes around the tumor were detected in ultrasound (arrows) 

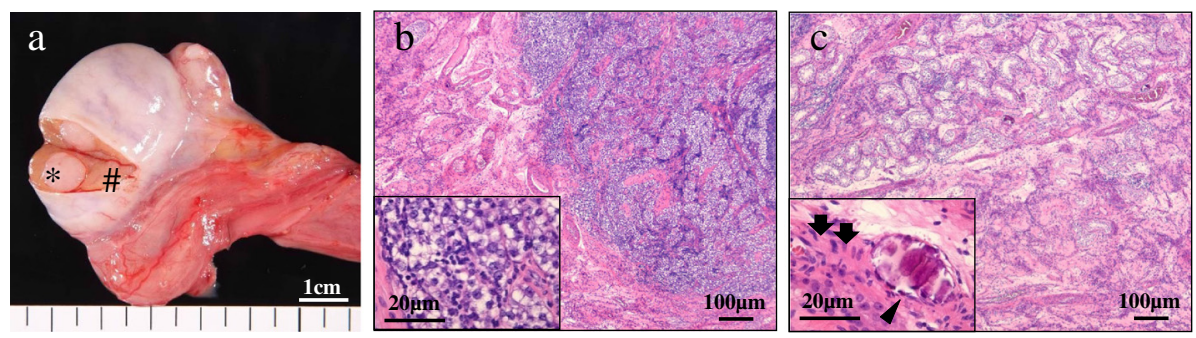

Fig. 2 a Macroscopic findings of orchiectomy specimen. A tiny yellowish tumor was observed (*), surrounded by normal tissue (\#). b Microscopic findings of the tiny tumor. Pathological findings revealed a pure seminoma. c Microscopic findings of the tissue around the tumor: calcification lesions (black arrowhead) and fibrous structures (black arrows)

with embryonal carcinoma [11]. In our case, before orchiectomy, our patient's DUPAN-2 level was higher than the measurable upper limit and it decreased to within the normal range after chemotherapy induction, suggesting that DUPAN-2 in our patient was produced by a nonseminomatous component like an embryonal carcinoma.

Seminomas typically appear as solid, round, homogeneous, low reflective masses contained within testes, without calcification inside the tumors. On the other hand, embryonal carcinomas and teratomas appear as inhomogeneous masses with calcification inside the tumor mass and, generally, a normal gonadal stroma does not contain calcifications. Miller et al. reviewed 3854 testicular ultrasound examinations; they hypothesized that intratesticular microcalcifications outside the margin of the tumor might represent a phenomenon of burned-out tumor [12]. In our case, an ultrasound examination revealed rough microcalcifications around the intratesticular tumor; in the orchiectomy specimen (Fig. 1c), many calcifications and fibrous tissues were detected around the seminomatous tumor (Fig. 2c). Those findings suggest that the testicular mass might result from a burned-out phenomenon of an embryonal carcinoma. Therefore, we performed immunohistochemical analyses for DUPAN-2 and AFP on the orchiectomy specimen and retroperitoneal mass. Immunohistochemical analyses showed no positive cells for DUPAN-2 or AFP in the orchiectomy specimen and retroperitoneal mass (Fig. 3). If we had obtained a biopsied retroperitoneal specimen in the first diagnosis, we might have been able to detect positive cancer cells.

In conclusion, here we report a case of pure testicular seminoma with elevated serum AFP and DUPAN-2 presenting retroperitoneal metastasis. We recommend an ultrasound examination of bilateral testes when large retroperitoneal tumors are detected in young men, even if a mass is not palpable in the scrotum.
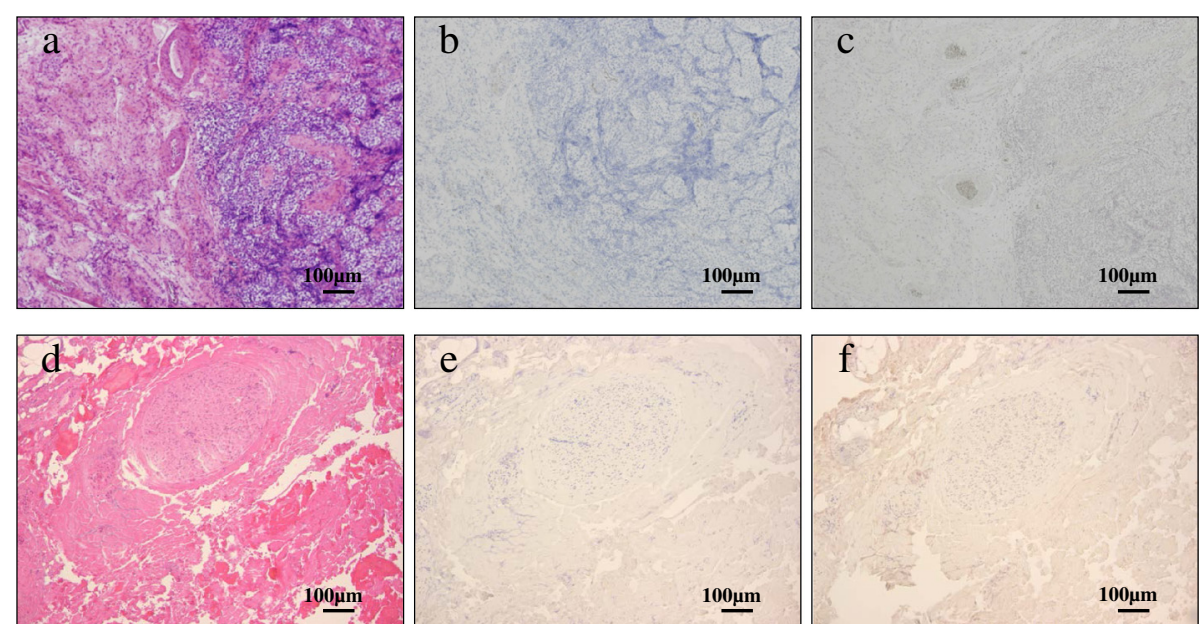

Fig. 3 Hematoxylin and eosin and immunohistochemical staining of the orchiectomy specimen $(\mathbf{a}, \mathbf{b}, \mathbf{c})$ and of the retroperitoneal dissected specimen (d, e, f). b and $\mathbf{e}$ alpha-fetoprotein staining; $\mathbf{c}$ and $\mathbf{f}$ DUPAN-2 staining 


\section{Conclusions}

We report a case of pure testicular seminoma at orchiectomy with elevated serum AFP and DUPAN-2 presenting retroperitoneal metastasis. When a retroperitoneal mass is detected and a testicular tumor is not palpable, we recommend consideration of the possibility of a testicular tiny tumor or of a burned-out phenomenon.

\section{Consent}

Written informed consent was obtained from the patient for publication of this case report and any accompanying images. A copy of the written consent is available for review by the Editor-in Chief of this journal.

\section{Abbreviations}

AFP: alpha-fetoprotein; BEP: cisplatin, etoposide, and bleomycin; CT: computed tomography; EP: etoposide and cisplatin; hCG: human chorionic gonadotropin; LDH: lactate dehydrogenase; NSGCTs: nonseminomatous germ cell tumors; RPLND: retroperitoneal lymph node dissection.

\section{Competing interests}

The authors declare that they have no competing interests.

\section{Authors' contributions}

Each author participated sufficiently in the work to take public responsibility for appropriate portions of the content. SI diagnosed this case and drafted the manuscript. TNai critically revised the manuscript. NK, RA, TE, Kl, TNag, $A O, K T$, and $Y S$ carried out the acquisition of data, coordination, and helped to draft the manuscript. TY supervised this manuscript. All authors read and approved the final manuscript.

\section{Author details}

'Department of Nephro-urology, Graduate School of Medical Sciences, Nagoya City University, 1, Kawasumi, Mizuho-cho, Mizuho-ku, 467-8601 Nagoya, Japan. ${ }^{2}$ Department of Pharmacy, Nagoya City University Hospital, Nagoya, Japan.

Received: 12 March 2016 Accepted: 21 April 2016

Published online: 05 May 2016

\section{References}

1. Motzer RJ, Agarwal N, Beard C, Bolger GB, Boston B, Carducci MA, et al. NCCN clinical practice guidelines in oncology: testicular cancer. J Natl Compr Canc Netw. 2009;7(6):672-93.

2. Oldenburg J, Fossa SD, Nuver J, Heidenreich A, Schmoll HJ, Bokemeyer C, et al. Testicular seminoma and non-seminoma: ESMO Clinical Practice Guidelines for diagnosis, treatment and follow-up. Ann Oncol. 2013;24 Suppl 6:vi125-32. doi:10.1093/annonc/mdt304.

3. Kundu SD, Carver BS, Sheinfeld J. Retroperitoneal histologic findings of patients with elevated serum alpha-fetoprotein and pure seminoma at orchiectomy. Urology. 2011;78(4):844-7. doi:10.1016/j.urology.2011.02.002.

4. Nazeer T, Ro JY, Amato RJ, Park YW, Ordonez NG, Ayala AG. Histologically pure seminoma with elevated alpha-fetoprotein: a clinicopathologic study of ten cases. Oncol Rep. 1998;5(6):1425-9.

5. Peterson M, Beck S, Bihrle R, Einhorn L, Foster R. Results of retroperitoneal lymph node dissection after chemotherapy in patients with pure seminoma in the orchidectomy specimen but elevated serum alpha-fetoprotein. BJU Int. 2009;104(2):176-8. doi:10.1111/j.1464-410X.2009.08697.x.

6. Herr HW, Sheinfeld J, Puc HS, Heelan R, Bajorin DF, Mencel P, et al. Surgery for a post-chemotherapy residual mass in seminoma. J Urol. 1997;157(3):860-2.

7. Masterson TA, Carver BS, Abel EJ, Pettus JA, Bosl GJ, Sheinfeld J. Impact of age on clinicopathological outcomes and recurrence-free survival after the surgical management of nonseminomatous germ cell tumour. BJU Int. 2012;110(7):950-5. doi:10.1111/j.1464-410X.2012.10947.x.
8. Metzgar RS, Gaillard MT, Levine SJ, Tuck FL, Bossen EH, Borowitz MJ Antigens of human pancreatic adenocarcinoma cells defined by murine monoclonal antibodies. Cancer Res. 1982;42(2):601-8.

9. Fujita K, Itoh Y, Wada R, Nagao K, Fujime M. Embryonal carcinoma producing DU-PAN-2 with burned-out phenomenon in the testis. Int J Urol. 2006;13(4):473-5. doi:10.1111/j.1442-2042.2006.01328.x.

10. Yamada Y, Tomita K, Fujimura T, Nishimatsu H, Takeuchi T, Kitamura T. Metachronous testicular tumor developing eight years after retroperitoneal extragonadal germ cell tumor. Int J Urol. 2008;15(3):267-9. doi:10.1111/j. 1442-2042.2007.01971.X.

11. Kamoshida S, Satoh Y, Yasuda M, Kajiwara H, Tsukinoki K, Osamura RY. Immunohistochemical heterogeneity of type 1 blood group antigen expressions in testicular germ cell tumors. Oncol Rep. 2002;9(4):845-51.

12. Miller FN, Rosairo S, Clarke JL, Sriprasad S, Muir GH, Sidhu PS. Testicular calcification and microlithiasis: association with primary intra-testicular malignancy in 3,477 patients. Eur Radiol. 2007;17(2):363-9. doi:10.1007/ s00330-006-0296-0.
Submit your next manuscript to BioMed Central and we will help you at every step:

- We accept pre-submission inquiries

- Our selector tool helps you to find the most relevant journal

- We provide round the clock customer support

- Convenient online submission

- Thorough peer review

- Inclusion in PubMed and all major indexing services

- Maximum visibility for your research

Submit your manuscript at www.biomedcentral.com/submit
Biomed Central 